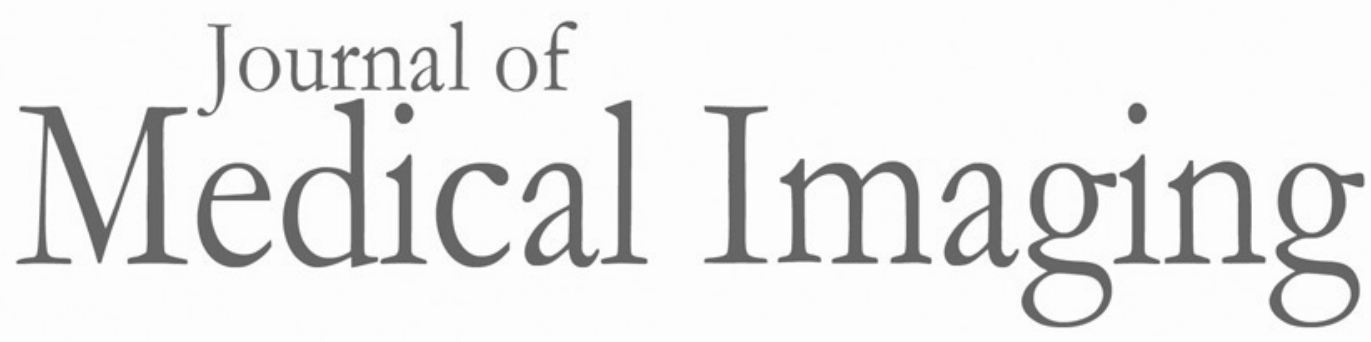

Medicallmaging.SPIEDigitalLibrary.org

\title{
Patient radiation exposure and dose tracking: a perspective
}

Madan M. Rehani SPIE. Madan M. Rehani, "Patient radiation exposure and dose tracking: a perspective," J. Med. Imag. 4(3), 


\title{
Patient radiation exposure and dose tracking: a perspective
}

\author{
Madan M. Rehani $i^{a, b, *}$ \\ aMassachusetts General Hospital and Harvard Medical School, Boston, Massachusetts, United States \\ bDuke University, Radiology and Medical Physics Department, Durham, North Carolina, United States
}

\begin{abstract}
Much of the emphasis on radiation protection about 2 decades ago accrued from the need for protection of radiation workers and collective doses to populations from medical exposures. With the realization that individual patient doses were rising and becoming an issue, the author had propagated the concept of a smart card for radiation exposure history of individual patients. During the last 7 years, much has happened wherein radiation exposure and the dose history of individual patients has become a reality in many countries. In addition to dealing with overarching questions, such as "Why track, what to track, and how to track?," this review elaborates on a number of points such as attitudes toward tracking, review of practices in large parts of the world, description of various elements for exposure and dose tracking, how to use the information available from tracking, achievements and stumbling blocks in implementation to date, templates for implementation of tracking at different levels of health care, the role of picture archiving and communication systems and eHealth, the role of tracking in justification and optimization of protection, comments on cumulative dose, how referrers can use this information, current provisions in international standards, and future actions. $\odot 2017$ Society of Photo-Optical Instrumentation Engineers (SPIE) [DOI: 10.1117/1.JMI.4.3.031206]
\end{abstract}

Keywords: patient radiation history; smart card; exposure and dose tracking; patient dose tracking; cumulative dose tracking. Paper 17045SSVR received Mar. 7, 2017; accepted for publication Jul. 10, 2017; published online Jul. 25, 2017.

\section{Introduction}

In the past, population dose was an important factor for policymaking in radiation protection. The fraction of the dose to population that comes from different imaging modalities, such as computed tomography (CT) had an important place. The advent of multidetector computed tomography (MDCT) was a turning point not only for clinical imaging but also for radiation protection. It has necessitated a shift of focus from collective dose to the population from medical exposures to individual patient dose. ${ }^{1}$ The MDCT made slice-by-slice scanning with interstice gap a thing of the past. Thinner slices with overlap and faster scanning that covered a larger area of the body, such as chest-abdomen-pelvis, increased the individual patient dose. Multiphase scanning became more common. In recent years, reports of overutilization of CT have emerged from the USA. ${ }^{2}$ All these contribute to the need for tracking patient exposures.

The word tracking has been used to represent different situations, and this needs to be clarified. Essentially, tracking can fall into two categories, namely (a) for an individual patient or (b) for a group of patients. Furthermore, tracking can be of the number and type of radiological procedures an individual patient has undergone or of the doses of these examinations or both. ${ }^{3}$

Regarding terminology, initially, there was a lack of clarity in use of the terms 'exposure' and 'dose' concerning tracking. There is now a better understanding on this. When a patient undergoes multiple radiological examinations, accounting for all the examinations, but without any consideration of dose,

*Address all correspondence to: Madan M. Rehani, E-mail: madan.rehani@ gmail.com this is referred to as exposure tracking. When the dose of each examination is also recorded, then this is termed dose tracking. ${ }^{4}$

In addition to overarching questions such as "Why track, what to track, and how to track?," this paper discusses themes, such as attitudes toward tracking, review of practices in large parts of the world, description of various elements for exposure and dose tracking, how to use the information available from tracking, templates for implementation of tracking at different levels of health care, the role of picture archiving and communication systems (PACS) and eHealth, the role of tracking in justification and optimization of protection, comments on cumulative dose, how referrers can use this information, what dose indices to track, the role of smart cards, current provisions in international standards, and future actions.

Since the tracking of radiological examinations and dose is gaining momentum, the motivation for this review article is to delve into this topic in its wider context to review the current status of tracking with regard to radiation protection of patients.

\section{Tracking of Radiological Procedures and Dose of an Individual Patient}

Tracking of radiological procedures of an individual patient is useful for individual patient protection as it provides clinical information that can avoid performing another radiological examination for that patient. Avoiding a repeat examination provides $100 \%$ dose reduction even when no account is made of dose from the previous examination.

Tracking of the dose of an individual patient is a concept that was established by the author through his work at the International Atomic Energy Agency (IAEA) ${ }^{5,6}$ and led to the

$1934-2608 / 2017 / \$ 25.00$ (C) 2017 SPIE 
Smart Card project of the IAEA. ${ }^{7}$ It received wide attention globally and has been implemented in imaging through PACS. Many European countries have implemented this, and experience from one country (Finland) that documented the impact of tracking in strengthening the process of justification and optimization of the individual patient has been presented. ${ }^{8}$ Individual dose tracking helps in optimizing follow-up examination as the patient acts as his/her own standard or reference for comparison in due consideration of clinical indication and body part. The approach on optimization in previous years has been based on comparing average values of dose [such as computed tomography dose index (CTDI)/dose-length product (DLP)] of a facility with diagnostic reference level (DRL) values. The DRL value is taken as a standard value. DRL has a mix of body weights and is not valid for an individual patient. Templates for use by countries at different levels of development have been provided. ${ }^{4}$ Worldwide needs were assessed, and results have been published. ${ }^{9}$ Furthermore, a survey of availability and use of unique patient identification numbers (ID) in 40 countries around the world was studied. ${ }^{10}$ Challenges for tracking of radiation doses from nuclear medicine examinations have been presented. ${ }^{11}$

Even though actions on tracking individual patient exposures have been pursued by the author of this paper since 2004, the first publicly available attempt to draw attention to cumulative radiation exposure to an individual patient by the author was in 2009 through an article in a nonindexed publication ${ }^{5}$ that received wide attention in the public and scientific media. ${ }^{12-14}$ It drew attention to the increasing individual patient doses wherein CT was becoming a main contributor. It envisaged individual patient dose becoming a tool for directing actions on patient protection and a shift in focus from occupational protection to patient protection.

An article by Sodickson et al. ${ }^{15}$ reviewed the number of CT scans over the past 22 years in a cohort of 31,462 patients. In all, $33 \%$ of patients underwent five or more lifetime CT examinations, and 5\% underwent between 22 and 132 examinations. About $15 \%$ of patients received estimated cumulative effective doses of $>100 \mathrm{mSv}$, and $4 \%$ received between 250 and $1375 \mathrm{mSv}$. The authors concluded that the cumulative CT radiation exposure added incrementally to the baseline cancer risk in the cohort, and this subgroup is potentially at higher risk due to recurrent CT imaging. This was followed by a number of papers in subsequent years assessing multiple CT scans that a patient undergoes in different clinical conditions and reporting doses of a few tens of $\mathrm{mSv}$ or even $100 \mathrm{mSv}$ that were received by the patient. ${ }^{16,17}$

\section{Tracking of Radiation Doses of a Group of Patients}

Tracking of radiation doses of a group of patients has become very prevalent in recent years. Many vendors have developed systems that provide dose information for every examination. This provides access to dose information, which helps in establishing DRLs, in comparing doses from time to time in the same institution, in making comparisons intrainstitution in different rooms to support the process of optimization. In previous years, it was common to establish the frequency of different radiological examinations and estimate collective dose to the population using the typical value of dose per examination. In addition, tracking of doses in a group of patients has been practiced by anonymizing patient data and results in patient dose registries. ${ }^{18,19}$ This helps in comparing one's own facility doses in any examination with dose with other facilities, which promotes optimization and can become a helpful tool in the accreditation process.

\section{Analysis of Attitudes Toward Tracking of Radiological Exposures and Dose}

When the author started talking about developing mechanisms for radiological exposure history in 2004, the reaction of most colleagues was invariably negative. An analysis of attitudes revealed several distinct reservations

i. it will take decades to achieve it (practicability),

ii. why do it? (purpose),

iii. the detection of overdosing of patients has implications relating to the responsibility of individual staff members (avoiding transparency), and

iv. there is no dose limit for patients, and thus how to make use of the cumulative dose (perceived notions in absence of clarity).

It is well known that managing any situation when the facts are known is better than managing it without any concrete information. It is widely taught that diagnosis is revealed in the patient's history. "Listen to your patient, they are telling you the diagnosis" is a much-quoted aphorism. ${ }^{20}$ Thus, looking into previous radiological examinations for clinical information is something that is natural but often missed in view of nonavailability or lack of easy access. It is here that specific concepts like that of tracking help. The concept has now reached the level of programs and gives impetus to solve traditional problems. On the issue of practicality (first point in the list above), it is amazing how many technological developments have occurred during the last decade. It was not possible in 2004 to think that listing of all examinations a patient has undergone will become possible with the click of a button in a few years. PACS systems were not talking to each other, although much had happened since 1995 through formal acceptance of Digital Imaging and Communications in Medicine (DICOM) standards. A high degree of variability of adoption of DICOM standards was responsible for integration difficulties. However, technology moved much faster than anticipated, probably due to the cancer risks from CT and impetus provided by a series of overexposures from CT scans during 2008 to 2009 with significant media attention where issues connected with safety became important. ${ }^{21,22}$ Now, it is a reality in most PACS to be able to list all examinations against a patient ID along with respective images and even the dose in most cases. The second point of "why track" is discussed further later in this paper in relation to justification and optimization. The third point pertaining to transparency is a dynamic one. People get persuaded with time as transparency generates benefits of easy information that leads to reliability and legitimacy among the constituents. In recent decades, there has been a transparency rush. With regard to the fourth point, it took the author a long time to understand the need for mentioning procedure tracking (exposure) separately from dose tracking and delinking tracking from cumulative dose.

\subsection{Cumulative Dose and Decision-Making}

The common notion that the purpose of tracking is to estimate the cumulative radiation dose is not correct. The author, with a group from Finland, has demonstrated that tracking of 
procedures and dose, without consideration of cumulative dose, has helped in strengthening the process of justification and optimization for individual patients. ${ }^{8}$ This has been demonstrated by case reports of children in the Helsinki province where a PACS system covers 33 hospitals and a tracking system has been established. $^{8}$

Lifetime history of radiological examinations is an area that is yet to mature. Like in the case of occupation exposure, despite the availability of a lifetime record, what really matters is the 5-year slot. Likewise, from a clinical standpoint, what may be of use is the examination done in past 2 years as in the case of screening mammography or in the last 1 year for many other diagnostic examinations to aid in avoiding unnecessary examination. From the point of view of cumulative dose, with the current recommendation of no dose limit for a patient, it may make no sense. In the future, if the concept of dose constraints is accepted, there may be a value of cumulative dose. However, despite the fact that the risk of 10th CT examination is the same as of the first CT, apparently, the human mind does get affected by previous events. This was evident from the IAEA surveys among referring physicians from 28 countries wherein the vast majority $(71.7 \%)$ of physicians felt that being aware of the history of CT scans would always or mostly lead them to a better decision on referring patients for CT scans. ${ }^{23}$ The risk of not performing the examination should be part of decisionmaking. The risk of an airplane accident may be same when one is flying for the first time or the 10th time, but a recent airplane accident does create an impact on the mind and the number of passengers suddenly drops. It is always a difference between what should be and what actually is. Note that one needs to give importance to "what is," but one should take it into consideration while deciding on "should." This is why surveys are done to first know what the ground realities are and how they can and should be improved.

We have not yet studied the value of cumulative dose in the process of justification and optimization, and this is an area of future research. This separation of cumulative dose from other aspects of tracking is essential, and it brings necessary clarity.

Looking back, it is evident that it does require time to have clarity on new concepts and for people to accept a new idea until it gains momentum and reaches a stage of implementation. It was interesting to have the first-hand experience of confronting resistance to a new idea and seeing the important role media can play in speeding up actions.

\section{Global Survey on Perceived Need for Tracking}

We conducted the first-ever survey in 2010 that covered 76 countries including the six most populous countries and 16 of the 20 most populous ones. It addressed the availability of prior radiation tracking programs, the potential usefulness of such a program, and familiarity with IAEA efforts in this area. ${ }^{9}$

The survey ${ }^{9}$ showed that, although no country had yet implemented a patient exposure tracking program at a national level, there was increased interest in this issue. Eight countries (11\%) indicated that such a program is actively being planned, and three $(4 \%)$ stated that they have a program for tracking procedures only, but not for dose. Twenty-two (29\%) felt that such a program will be "extremely useful," 46 (60\%) "very useful," and eight (11\%) "moderately useful," with no respondents stating "mildly useful" or "not useful." Ninety-nine percent of countries indicated an interest in developing and promoting such a program. There were successful examples at the subnational level where tracking had become possible within a few dozen hospitals covered by PACS systems and there were nascent efforts to extend coverage nationwide. These findings serve as a benchmark and stimulus for future radiation exposure and dose recording efforts, which offer the potential to create a datarich environment that enables maximal global implementation.

The survey also included dose quantities that will be tracked. For projection radiography (x-ray, computed/digital/dental radiography), the most common choice was dose-area product (DAP)/kerma-area product (KAP) $(5 / 7=71 \%)$, whereas some also chose entrance surface air kerma $(2 / 7=28 \%)$, exposure index $(2 / 7=28 \%)$, organ absorbed dose, and effective dose.

For fluoroscopy, again, the most common choice was DAP/ KAP $(7 / 7=100 \%)$, but some participants also chose fluoroscopy time $(4 / 7=57 \%)$, cine time $(4 / 7=57 \%)$, cine runs $(2 / 7=28 \%)$, and/or dose/air kerma at the interventional reference point $(4 / 7=57 \%)$.

For CT, the common choices were volume CTDI $\left(\mathrm{CTDI}_{\mathrm{vol}}\right)$ $(7 / 7=100 \%), \quad \operatorname{DLP} \quad(6 / 7=86 \%)$, and weighted CTDI $\left(\mathrm{CTDI}_{w}\right)(2 / 7=28 \%)$; and one respondent also chose effective dose.

For mammography, three respondents only selected average glandular dose, whereas one also included incident air kerma.

For nuclear medicine, most selected administered activity (MBq) and organ absorbed dose; one also included effective dose.

Prior to 2006, when there was little international guidance on dosimetry in diagnostic radiology, there was a lack of clarity on what dose quantities to use in different imaging situations. The availability of guidance from ICRU and IAEA ${ }^{24,25}$ remedied the situation, and now there is a high degree of stability on reference dose quantities.

\section{Elements of Patient Exposure and Dose Tracking}

\subsection{Patient Identification Number}

The most crucial element for the success of this project is the availability of a unique patient ID that is used every time the patient undergoes a radiological examination. If the same ID is nationally used anywhere the examination is performed, the tracking can be achieved nationally, provided the network connects all imaging facilities. Not all countries have a permanent ID for every national or permanent resident, and, even if they have, they may not use it for medical record purpose. We conducted a survey to assess the availability and use of IDs in less-resourced countries. ${ }^{10}$ Forty countries responded. It was highly encouraging to note that a unique ID was available in 33 of 40 countries $(82.5 \%)$ and is used in the medical record and for tracking of imaging examinations in 18 of the 33 $(54.5 \%)$ countries. Furthermore, nearly one half of respondents indicated that lack of technology rather than confidentiality was the reason for not using a patient ID. Nearly one third of the countries-all 12 in Europe - supported the need for regulations requiring the tracking of patient radiation exposure.

One can wonder how tracking of individual patient exposure can be made possible within and across different states in the USA where confidentiality issues occupy a supreme position and the patient information needs to be deidentified. A similar situation will apply to any country where patient ID cannot be maintained during communication of medical imaging studies. 


\subsection{Role of Smart Cards}

The smart card is usually a card with a built-in microprocessor, typically used for electronic processes, such as financial transactions and personal identification. Initially, when we launched the IAEA Smart Card project, ${ }^{7,26}$ the common impression it generated, despite our intentions not being so, was that the card will contain radiation dose data for the patient. This impression took a long time to dispel by giving examples of ATM card and credit card, which do not have cash or credit on the card, but one can access cash or credit using the card as a digital signature. The data are available on servers that may maintain a dose registry. Similarly, the smart card for radiation exposure tracking, which was subsequently named as smart card/SmartRadTrack, is meant to provide access to data on servers. The servers currently are PACS servers; in the future, these may be dose repositories or registries that may not deidentify patients. The developments in eHealth, for example, the European Commission's eHealth Action Plan 2012 to 2020, provide a roadmap to empower patients and health-care workers. They link devices and technologies and invest in research toward the personalized medicine of the future. ${ }^{27}$

If a patient chooses to have an eHealth record, they consent to health-care providers accessing and uploading clinical documents. Further details on data protection, information governance, and patient consent are beyond the scope of this article and are governed by policies under eHealth. This ability to set access control measures is a key privacy feature of the eHealth record system.

When health professionals with authorized access in a facility just key in the patient ID and access information, one can obviate the need for a smart card.

\subsection{Dose Servers}

There are systems available that draw dose and patient data without images and transport them to radiation monitoring servers. ${ }^{22}$ Medical physicists normally require access to dose information without images, and thus the system caters to this need. Currently, a list of all radiological examinations pertaining to the patient in the server of the network is easily obtained. A click on an examination provides dose information with the capability of also accessing images if this is built into the system. This information can be available as a file that can be communicated further. The system is usually capable of extending outreach to wherever the radiation exposure monitoring server extends, at the county, state, or national levels. In addition, the access can be extended through hospital information system (HIS) or eHealth system to referrers. The patient record, once open, can be queried and researched for details of the patient's radiation dose history if desired. Further details about a prototype are available. ${ }^{28}$

\subsection{Data Storage and Access to Full Data Within Imaging Facilities}

There are a number of possibilities for keeping complete data records including images on servers that can be accessed through the PACS network or by integration with the radiology information system (RIS) or HIS. The most feasible is the one integrated into PACS on the same server. This does not usually require additional software as most modern PACS have the necessary software to enable a display of all examinations for a patient at locations within the PACS network, even in different cities within the same country.

Whenever the examination is performed outside the network of hospitals connected by the PACS where access is made, and if the patient mentions examinations performed in another part of the country, images and related information can be obtained by sending a request to the PACS administrator in the other region. This will normally require time and connection between the two PACS for the requested information as the two systems are not always communicating live. Such systems are in existence in many European countries who have implemented regional (subnational) PACS and some national PACS.

\section{Use of Radiation Dose Data}

After achieving tracking of exposures and dose, the next question that arises is how to use this information. Imaging modalities deliver dose data in different quantities, mostly in reference dose quantities, such as CTDI ${ }_{\mathrm{vol}}$ and DLP in CT and KAP and cumulative air kerma in the case of fluoroscopy. These dose quantities can be used by experts, such as medical physicists, to consolidate dose information from various imaging modalities, convert them into effective dose, and communicate them to radiologists and clinicians as dose and risk estimates with due caution. It is the responsibility of the medical physicist to handle information in different units. It is not desirable for imaging systems to provide dose information in terms of effective doses, which normally have large variabilities and inaccuracies. Professionals not versed in these inaccuracies may overuse and misinterpret figures. A paper from our group has demonstrated how dose information of an individual patient from different imaging studies was useful in identifying scope for optimization. ${ }^{8}$ In a previous paper, ${ }^{4}$ we have provided a comprehensive list of potential benefits from patient exposure tracking, which is reproduced here in Table 1. These benefits include those to patients, to health-care providers who refer patients and who perform imaging studies, and to policymakers, regulators, and researchers.

\section{Role of Referrers}

The referrers play a role in evaluating and comparing images with previous studies to contribute to the justification of radiological examination. This requires access through HIS, RIS, or eHealth systems. There are access control mechanisms usually in place to allow or forbid access to specified information. Invariably the referrer will need access to previous images, together with interpretation by an imaging professional, rather than dose. These days, there are possibilities for providing access for referrers through virtual private networks and intranet on mobile phones that facilitates decision-making to allow images to accompany the report from the radiation specialist. It is hoped that there will be increasing use of clinical information available in previous imaging studies, obviating the need for many repeat studies; thereby, tracking of procedures helps in strengthening the process of justification.

As indicated above, at this moment, it is not possible to provide guidelines on how referrers can use dose history for making judgments about subsequent examinations. With a lack of understanding about dose on part of referrers, there can be dangers in misinterpreting dose figures. Furthermore, there has been opposition to the use of cumulative dose received by the patient as part of the justification of the next examination. While such arguments may be valid in clear-cut situations, so-called black 
Table 1 Potential benefits from patient radiation exposure tracking. ${ }^{4}$

I. Benefits to patients

a. Receive minimal radiation exposure needed for optimal care

b. Knowledge that there is accountability/responsibility in the delivery of medical radiation

c. Facilitate dialogue with health-care providers regarding radiation exposure

d. Improve patient confidence in health-care providers' care

II. Benefits to health-care providers referring patients for imaging/intervention
a. Improved justification including decision support
b. Control resources/costs from unneeded duplicate tests
C. Minimize radiation effects by tracking cumulative exposure
d. Assist in choosing among imaging/intervention providers and facilities
e. Assist in choosing between modalities and techniques
f. Facilitate dialogue with patients regarding radiation exposure
g. Improve patient confidence in health-care providers' care

III. Benefits to health-care providers involved in performance of imaging/intervention
a. Improved justification including decision support
b. Control resources/costs from unneeded duplicate tests
c. Minimize radiation effects by tracking cumulative exposure
d. Assist in protocol optimization
e. Establishment and continuous review of reference levels
f. Provides dosimetric feedback mechanism for health-care provider quality improvement
g. Facilitate dialogue with patients regarding radiation exposure
h. Improve patient confidence in health-care providers' care

IV. Benefits to policymakers
a. Improved quantitative tools to protect the public health and safety
b. Improved quantitative approaches to radiation safety policymaking
c. Control resources/costs from unneeded duplicate tests

V. Benefits to regulators
a. Establishment and continuous review of reference levels
b. Provide data-rich regulatory environment enabling assessment of practice patterns beyond a single reference level
c. Ability to quantitatively audit individual providers, practices, and facilities

VI. Benefits to researchers
a. Provide extensive and robust radiation safety data sets to address research questions
b. Incorporate patient-specific radiation metrics into research studies, including observational, epidemiological, comparative effectiveness, outcomes, and randomized control
c. Provides quantitative basis for development of best practices
d. Incorporate radiation metrics into appropriateness criteria

VII. Benefits to industry

a. Facilitate partnership with other stakeholders in establishing radiation cumulative exposure programs 
and white situations, there are a large number of situations where there are many shades of gray and it is not a clear-cut yes-no situation. Any amount of additional information can be very helpful, as is evident from the first-ever multinational survey among referrers that IAEA conducted. ${ }^{23}$ The aim was to survey opinions of referrers on the importance of acquiring information about previous diagnostic exposures. ${ }^{23}$ Seven hundred and twenty-eight referrers from 28 countries responded (52.3\% from developed and $47.7 \%$ from developing countries). The vast majority $(71.7 \%)$ of physicians feel that being aware of the previous history of CT scans would always or mostly lead them to a better decision on referring a patient for a CT scan, but only $43.4 \%$ often enquired about it. The majority of referrers $(60.5 \%)$ stated that having a system that provides quick information about patient exposure history would be useful.

In future years, this issue needs to be further explored and debated.

\subsection{Communication with the Patient}

Normally, clinicians are at the forefront in communicating with patients, and they seek information from radiological professionals or in modern times through the Internet. Invariably, clinicians are not familiar with radiation dose quantities and their appropriate use. Medical physicists, radiologists, and radiological professionals need to create awareness on how to make sense of the information provided through tracking and make explanations available through the Internet, which unfortunately is not the case currently. Currently, the practice is reasonably developed for the use of effective dose as a risk metric, notwithstanding its overuse, but less for the use of organ dose. Medical physicists should take into account radiation doses from different radiological examinations, calculate effective dose or organ doses as appropriate, and provide meaningful information that can be communicated to the patient. Providing dose figures to the patient at the first instance may lead to potential misinterpretation. There is a lot to be done in terms of the development of communication strategies for dose information that is pooled from different imaging studies.

\section{Templates for Implementation of Patient Radiation Exposure or Dose Tracking}

There being wide variations in the global situation and increasing interest in this topic, we have previously provided templates that deal with different elements for implementation of exposure and dose tracking. ${ }^{4}$ We deliberated on various levels, such as practice, multipractice, national, and international. A template for less-resourced countries was also addressed. At the practice level, one needs primarily patient ID and a PACS system that connects all radiological imaging systems from all vendors within the practice with the capability to display all examinations of a patient when the patient ID is entered. At the multipractice level, PACS-interconnected hospitals must use the same patient ID with full connectivity including structured dose reports. Implementation at the national level is a matter of extension of principles from county/state level to national level. There are increasing issues of access controls needed when larger networks are encountered.

\section{Nuclear Medicine Exposures}

Tracking mechanisms rely on the availability of structured dose reports, typically found in the DICOM header. While dosimetric information is currently readily available for many individual $\mathrm{x}$-ray-based procedures, unfortunately, nuclear medicine, which constitutes a large part of radiation exposure to the patient population, currently lags behind x-ray-based procedures with respect to reporting of radiation dosimetric information electronically in patient files that are transmitted over a network. Currently, in nuclear medicine studies, one can include mention of administered activity. There is no system to give the dose to a representative phantom, if not to a patient. This is not to the same level as x-rays where the dose to a representative phantom is provided in a patient file based on the exposure factors employed. It is all the more important in hybrid imaging [positron emission tomography (PET)/CT and single-photon emission computed tomography (SPECT)/CT] where the doses from the PET and SPECT part are substantial. A paper by our group ${ }^{11}$ discussed qualitative differences between nuclear medicine and x-ray-based procedures, including differences in the radiation source and measurement of its strength, the impact of biokinetics on dosimetry, and the capability of current scanners to record dosimetric information. These differences depict challenges in applying monitoring and reporting strategies used in x-ray-based procedures to nuclear medicine and integrating dosimetric information across modalities. The development of strategies to improve the reporting of radiation dosimetric data in nuclear medicine provides a challenge for the future. Some ideas on how to address this issue were suggested in that paper and are not repeated here.

\section{Consideration of Radiotherapy Exposures}

In radiotherapy, the dose is deliberately delivered to kill or modify selected cells, unlike diagnostic exposures where the purpose is to obtain image and radiation exposure happens to be a part of the selected examination. When the purpose of tracking is to improve or strengthen the process of justification and optimization of patient protection in diagnostic studies, radiotherapy dose is not included in tracking. In the future, when practices get developed and there are models for better considerations of doses to organs not only for risk estimates but also for utilization of nontarget organ doses, one could think of the inclusion of that dose in tracking.

\section{Standards}

Basic Safety Standards (BSS) of IAEA require that justification of medical exposure for an individual patient shall be carried out and account taken of relevant information from that patient's previous radiological procedures, among other things. ${ }^{29}$ The European BSS $^{30}$ defines that "clinical responsibility" means responsibility of a practitioner for individual medical exposures, in particular, justification; optimization; clinical evaluation of the outcome; cooperation with other specialists and staff, as appropriate, regarding practical aspects of medical radiological procedures; obtaining information, if appropriate, on previous examinations; providing existing medical radiological information and/or records to other practitioners and/or the referrer, as required; and giving information on the risk of ionizing radiation to patients and other individuals involved, as appropriate. Furthermore, the referrer and the practitioner, as specified by the member states, seek, where practicable, to obtain previous diagnostic information or medical records relevant to the planned exposure and consider these data to avoid unnecessary exposure. Thus, there is no doubt that there are requirements on using diagnostic information from previous examinations. There is 
no explicit mention of dose from previous examinations. To date, it has been left out in view of the absence of any dose limit for patients and no attempt has been made to provide guidelines when the individual patient dose has reached the level that we have today, for some patients. As a result, physicians are left with decision-making based on gut feelings, which explains the response to the survey among referrers. ${ }^{4}$ There is an urgent need to provide guidelines and clarity in standards to help in such situations.

\section{Future}

With increasing access to radiological imaging, in particular, CT in many developing countries, it appears that this topic is going to become all the more important in coming years. In developed countries, where the problems pertain to overutilization of $\mathrm{CT}$, such as in the USA, ${ }^{2}$ tracking can be helpful not only in reducing the number of $\mathrm{CT}$ examinations but also in reducing inappropriate CT examinations through enhanced individual justification. ${ }^{4,6,8}$

There will be a need in certain countries, where there has been a more rapid and less controlled expansion of the use of CT, to develop guidelines for patients who have received high doses of a few tens of $\mathrm{mSv}$ or a $100 \mathrm{mSv}$ of effective dose where they need further imaging studies. The existing concept of a dose constraint that is currently not applicable to patients may need to be introduced. This will be yet another new step that will require crossing hurdles. The author has raised this issue within International Commission on Radiological Protection, but there is a lot of resistance, as is typical of anything that requires a change in the established frameworks. On the issue of cumulative dose, there have been considerations relating to the risk of radiation-induced carcinogenesis for utilization strategies. ${ }^{31}$ In another paper focusing on patient-centric actions, it is recommended that optimal methods to translate cumulative patient-centric dose to risk of malignancy need to be developed and validated to present to physicians and patients for adequate risk-benefit analysis in the context of decisions for further imaging in individual patients. ${ }^{32}$ Such risk analysis could similarly inform surveillance strategies in patients already exposed to some level of cumulative radiation dose. An action plan was developed by the "think tank" for cardiovascular imaging that recommended, in addition to others, robust dose assessment and longitudinal tracking. ${ }^{33}$

Tracking will provide useful dose data for future epidemiological studies on patients who have been exposed to tens of $\mathrm{mGy}$ doses to organs or a few tens of $\mathrm{mSv}$ of effective dose, particularly during childhood.

\section{Summary}

There are very few examples where so much has been achieved in so few years as in the case of patient exposure tracking. Some countries in Europe already have the capability of tracking radiological examinations and doses to an individual patient conducted nationwide. Many European countries are on the way to nationwide PACS systems that will instantaneously make tracking possible if the country uses permanent patient ID. The European Commission's plans for cross-border health care will cross hurdles of confidentiality, data protection, access controls, information governance, and patient's consent. It has been documented that patient-specific justification and optimization becomes possible using the tracking of radiological procedures and radiation dose of individual patients. There are many challenges that need to be addressed where future work will be needed, e.g., exploring utilization of cumulative dose in the context of decision-making for the next examination, the possibility of dose constraints for patients, and the extent to which referrers should be concerned with doses from examination. Tracking will provide useful dose data for future epidemiological studies on patients who have been exposed to tens of $\mathrm{mGy}$ doses to organs or a few tens of $\mathrm{mSv}$ of effective dose, particularly during childhood.

\section{Disclosures}

No conflicts of interest, financial or otherwise, are declared by the authors.

\section{Acknowledgments}

This perspective article is based on the publications and the work of the author during his tenure at the International Atomic Energy Agency (IAEA), Vienna, Austria. It contains review of other work in the area during recent years.

\section{References}

1. M. M. Rehani and E. Vano, "Radiation protection in medicine in next decade," Radiat. Prot. Dosim. 147, 52-53 (2011).

2. W. R. Hendee et al., "Addressing overutilization in medical imaging," Radiology 257(1), 240-245 (2010).

3. M. M. Rehani, "Tracking of examination and dose: overview," Radiat. Prot. Dosim. 165, 50-52 (2015).

4. M. Rehani, T. Berris, and D. P. Frush, "Templates and existing elements and models for implementation of patient exposure tracking," Radiat. Prot. Dosim. 158, 36-42 (2013).

5. M. M. Rehani, "Smart protection," IAEA Bull. 50(2), 1-3 (2009).

6. M. Rehani and D. Frush, "Tracking radiation exposure of patients," Lancet 376, 754-755 (2010).

7. M. M. Rehani and D. P. Frush, "Patient exposure tracking-the IAEA smart card project," Radiat. Prot. Dosim. 147, 314-316 (2011).

8. R. Seuri, M. M. Rehani, and M. Kortesniemi, "How tracking patients radiological procedures and dose helps?: experience from Finland," Am. J. Roentgenol. 200, 771-775 (2013).

9. M. Rehani et al., "Patient radiation exposure tracking: worldwide programs and needs-results from the first IAEA survey," Eur. J. Radiol. 81, e968-e976 (2012).

10. M. M. Rehani and T. Berris, "Survey of unique patient identification number in 40 countries for radiation exposure tracking," Am. J. Roentgenol. 200, 776-779 (2013).

11. M. Mercuri, M. M. Rehani, and A. J. Einstein, "The need for an integrated approach to tracking radiation exposure: challenges with nuclear medicine," J. Nucl. Cardiol. 19(5):895-900 (2012).

12. CNN Coverage, "IAEA calls for enhanced radiation protection of patients," 2009, http://www.youtube.com/watch?v=h-9x4y7hhWk (20 July 2017).

13. Diagnostic Imaging, "International agency wants smart cards to track patient radiation histories," 2009, http://www.diagnosticimaging.com/ articles/international-agency-wants-smart-cards-track-patient-radiationhistories (20 July 2017).

14. "News Links on Smart Card/SmartRadTrack project," 2009, https://rpop .iaea.org/RPOP/RPoP/Content/Documents/Whitepapers/smart-card-links .pdf (20 July 2017).

15. A. Sodickson et al., "Recurrent CT, cumulative radiation exposure, and associated radiation-induced cancer risks from CT of adults," Radiology 251, 175-184 (2009).

16. R. T. Griffey and A. Sodickson, "Cumulative radiation exposure and cancer risk estimates in emergency department patients undergoing repeat or multiple CT," Am. J. Roentgenol. 192, 887-892 (2009).

17. T. A. Jaffe et al., "Radiation doses from small-bowel follow-through and abdominopelvic MDCT in Crohn's disease," Am. J. Roentgenol. 189, 1015-1022 (2007).

18. V. A. Murugan et al., "American college of radiology dose index registry: a user's guide for cardiothoracic radiologists part 1: dose index 
registry (DIR)-what it means and does for CT?" J. Thorac. Imaging 30(6), W66-W68 (2015).

19. V. A. Murugan et al., "ACR DIR: a user's guide for cardiothoracic radiologists: part 2: how to interpret your DIR report," J. Thorac. Imaging 30(6), W69-W72 (2015).

20. R. Smith, "Thoughts for new medical students at a new medical school," Br. Med. J. 327, 1430-1433 (2003).

21. W. Bogdanich, Radiation Overdoses Point Up Dangers of CT Scans, The New York Times, New York, USA (2009).

22. M. M. Rehani, "What makes and keeps radiation risks associated with CT a hot topic?" Am. J. Roentgenol. 204(3), W234-W235 (2015).

23. M. M. Rehani and T. Berris, "International Atomic Energy Agency study with referring physicians on patient radiation exposure and its tracking: a prospective survey using a web-based questionnaire," BMJ Open 2, e001425 (2012).

24. International Commission on Radiation Units and Measurements, "Patient dosimetry for x-rays used in medical imaging," ICRU Report 74, Bethesda, Maryland (2005).

25. International Atomic Energy Agency, "Dosimetry in diagnostic radiology: an international code of practice," Technical Report Series 457, IAEA, Vienna (2007).

26. "IAEA Smart card/SmartRadTrack project," 2009, https://rpop.iaea.org/ RPOP/RPoP/Content/News/smart-card-project.htm (20 July 2017).

27. European Commission, "eHealth action plan 2012-2020: innovative healthcare for the 21st century," 2012, http://ec.europa.eu/digital-agenda/ en/news/ehealth-action-plan-2012-2020-innovative-healthcare-21stcentury (20 July 2017).

28. M. M. Rehani and J. F. Kushi, "A study on smart card for radiation exposure history of patient," Am. J. Roentgenol. 200, 780-782 (2013).

29. International Atomic Energy Agency, "Radiation protection and safety of radiation sources: international basic safety standards-Interim edition," in General Safety Requirements Part 3, IAEA Safety Standards Series GSR Part 3 (Interim), International Atomic Energy Agency (IAEA), Vienna (2011).

30. European Commission, "Council directive 2013/59/Euratom of 5 December 2013 laying down basic safety standards for protection against the dangers arising from exposure to ionising radiation, and repealing directives 89/618/Euratom, 90/641/Euratom, 96/29/Euratom, 97/43/Euratom and 2003/122/Euratom,” Off. J. Eur. Commun. L13, 1-73 (2013).

31. D. J. Durand, R. L. Dixon, and R. L. Morin, "Utilization strategies for cumulative dose estimates: a review and rational assessment," J. Am. Coll. Radiol. 9, 480-485 (2012).

32. A. Sodickson and R. Khorasani, "Patient-centric radiation dose monitoring in the electronic health record: what are some of the barriers and key next steps?" J. Am. Coll. Radiol. 7, 752-753 (2010).

33. P. S. Douglas et al., "Developing an action plan for patient radiation safety in adult cardiovascular medicine: proceedings from the Duke University Clinical Research Institute/American College of Cardiology Foundation/American Heart Association Think Tank held on February 28, 2011," J. Am. Coll. Radiol. 59(20), 1833-1847 (2012).

Madan M. Rehani is currently a director of Global Outreach for Radiation Protection at the Massachusetts General Hospital, Harvard Medical School, and an adjunct professor in radiology and medical physics at Duke University. Formerly, he is a radiation safety specialist at the International Atomic Energy Agency for 11 years and prior to that professor and head of the medical physics at the All India Institute of Medical Sciences, New Delhi, India. He is a member of the International Commission on Radiological Protection since 1997. He is an associate editor of The British Journal of Radiology. He has published 200 publications, and he is a vice president of IOMP. 